\title{
Computer Algebra Manipulations in Astronomy
}

\author{
Tamara Ivanova \\ Institute of Applied Astronomy \\ 191187, St.Petersburg, Russia \\ itv@quasar.ipa.nw.ru
}

\begin{abstract}
The specialized Poisson Series Processors (PSPs) are proposed. They are a typical software for the implementation of analytical algorithms of celestial mechanics. The PSPs are designed for manipulating long Poisson (polynomial-trigonometric) and echeloned series with the rational, floating-point or arbitrary length (infinite precision) coefficients and with unlimited number of power and angular variables. The Keplerian processor and analytical generator of special celestial mechanics functions based on the PSPs are also developed.
\end{abstract}

\section{Introduction}

The main objects of celestial mechanics are so-called Poisson series, i.e., the polynomial-trigonometric series of the form:

$$
\begin{gathered}
S=\sum C_{i, j} x_{\text {cos }}^{i \sin }(j y), \\
x=\left(x_{1}, \ldots, x_{n}\right), \quad y=\left(y_{1}, \ldots, y_{m}\right), \quad i=\left(i_{1}, \ldots, i_{n}\right), \quad j=\left(j_{1}, \ldots, j_{m}\right) .
\end{gathered}
$$

Here $x$ and $y$ are vectors of polynomial and trigonometric variables, respectively. The summation is performed over all integer values of indices $i$ and $j$. Coefficients $C_{i, j}$ may be represented as rational, floating-point, arbitrary length or complex numbers. The last form of coefficients corresponds to the exponential Poisson series:

$$
S=\sum C_{i, j} x^{i} \exp \sqrt{-1}(j y) .
$$

The systems of operations on the series of this kind are known as Poisson processors. These specialized processors turn out to be very effective in performing the symbolic operations with series even of hundreds of thousand terms characteristic of high-accuracy analytical theories of celestial mechanics. Though in general they are constructed for the purpose of solving celestial mechanics problems they may be used equally well in other fields such as theoretical mechanics, physics, etc. Poisson processors have been developed in many centres of celestial mechanics. The brief review of them and their applications to solve the celestial mechanics problems are given by Brumberg (Brumberg, 1995). As a rule they are different in the representation of coefficients, in the number of power and trigonometric variables, in the range of associated indices of these variables, in storage techniques for the Poisson series, etc. Some of them allow 
manipulation of a wider class of objects than the Poisson series, for instance, so-called echeloned series (very long polynomial-trigonometric series with literal frequencies):

$$
\begin{gathered}
S=\sum x^{i}{ }_{\cos }^{\sin }(j y) \sum_{k=1}^{K_{i, j}} C_{i, j, k} \prod_{\nu=1}^{N_{k}}\left(\gamma^{(\nu, k)} \omega\right)^{q^{(\nu, k)}}, \\
x=\left(x_{1}, \ldots, x_{n}\right), \quad y=\left(y_{1}, \ldots, y_{m}\right), \quad i=\left(i_{1}, \ldots, i_{n}\right), \\
j=\left(j_{1}, \ldots, j_{m}\right), \quad \omega=\left(\omega_{1}, \ldots, \omega_{m}\right), \quad \gamma^{(\nu, k)}=\left(\gamma_{1}^{(\nu, k)}, \ldots, \gamma_{m}^{(\nu, k)}\right) .
\end{gathered}
$$

Each term of this series is characterized by the set of power $x$, angular $y$ and frequency $\omega$ variables with associated multi-indices $i, j$ and $\gamma^{(\nu, k)}$ together with the quantities $q^{(\nu, k)}$, respectively. The coefficients $C_{i, j, k}$ are rational, floatingpoint or arbitrary length numbers. The exponents $q^{(\nu, k)}$ are integers. The first summation is again performed here over all integer values of indices $i$ and $j$. The values of $K_{i, j}$ and $N_{k}$ are directly obtained as a result of symbolic operations on the series.

In this case the trigonometric variables $y_{r}$ are represented by the linear functions of an argument $t$ with the literal values of the frequencies $w$ :

$$
y_{r}=w_{r} t+y_{r}^{(0)}, \quad r=(1, \ldots, m) .
$$

The Echeloned Poisson Series Processor EPSP (Ivanova, 2001) is designed for manipulating the echeloned series. This processor is developed on the basis of the Poisson series processor PSP (Ivanova, 1995). PSP is intended directly for operating on the Poisson series themselves, that is, the polynomial-trigonometric series where the frequencies are taken into account numerically in differentiating and integrating with respect to argument $t$. The echeloned series processor allows to solve a wider class of problems as compared with the Poisson series processor. This is a case, for instance, of analytical Lunar theory where one has to handle the divisors in symbolic form. From the user's point of view both processors are similar, except that the list of basic operations of EPSP includes the operations of the total differentiation and integration with respect to time in the analytical form relatively to the frequencies. It leads to more complex storage schemes in the echeloned series processor.

\section{Basic Characteristics of PSPs}

The most important characteristics of PSPs are as follows:

- PSPs are written in standard FORTRAN-77 language.

- PSPs have no restrictions on the number of power, angular and frequency variables and on the ranges of changing of associated indices to be prescribed by user for any specific problem. For comparison, processor ESP (Rom, 1971) deals with the echeloned series of 12 polynomial, six trigonometric and three frequency variables. 
- Every term of the series is characterized by the analytical order of smallness $N$ calculated by formula

$$
N=\sum_{l=1}^{n} i_{l} W_{l}
$$

where $W_{l}$ is the weight function of the polynomial variable with number $l, i_{l}$ is the corresponding index. The analytical order of smallness is a very important characteristic because the power variables are as a rule the small parameters resulting in the possibility of restricting the series by the terms with the total order of the power variables not more than some prescribed value.

- All mathematical operations with the series are formal. The criterion for rejecting a term is based on its smallness determined by the analytical order of the term, the numerical estimation of its coefficient and the admissible values of the indices.

- PSPs allow the user to write his/her own procedures without any difficulty. It is especially easy to perform the identical operations on every term of the series due to the availability of special scanning procedures executing a given action on each term of series.

- The hierarchical architecture of PSPs allow rather easily the adaptation of the system on different computers and the modification of it for the objects slightly different from the standard Poisson series (for instance, for manipulating the exponents instead of the trigonometric functions or for changing the type of coefficients) or in the case of using different storage techniques for series. The hierarchical principle involves the subdivision of all system procedures into three levels. The procedures of the lowest level realize the operations on coefficients of terms and their indices. The subroutines of the middle level realize the operations on individual terms of the series and depend on specific representation of Poisson series in computer memory. Finally, the analytical operations on the series are realized by the highest level procedures taking into account only general mathematical structure of objects.

- The list of basic operations of PSPs includes the standard arithmetic operations with the series, the partial differentiation and integration with respect to polynomial and trigonometric variables, the total differentiation and integration with respect to time, raising to any integer, rational or real exponent, binomial and Taylor expansion up to some prescribed order, substitution of the series in place of any set of the power and trigonometric variables, fast evaluation of the series for numerical values of any variables, conversion of the series, different sortings and selections, etc. PSPs enable the realization of the input and output of the series in any format or unformatted mode and typing them in natural mathematical form.

All algorithms of the operations with the series imply the standard lexicographic order of the input series and do not alter it in the resulting series. 


\section{Storage Schemes of PSPs}

The storage area consists of two or three global arrays which keep the series terms, their coefficients and divisors or factors for the EPSP. The memory for coefficients is represented by the standard one-dimensional array where rational, floating-point and complex coefficients are located consecutively taking into account their length depending on their type. Two other arrays for keeping the series, divisors and factors and first array for arbitrary length coefficients use the one-way linked list structure for dynamical memory allocation (Figure 1) permitting the application of an algorithm for quick searching and inserting of terms into series.

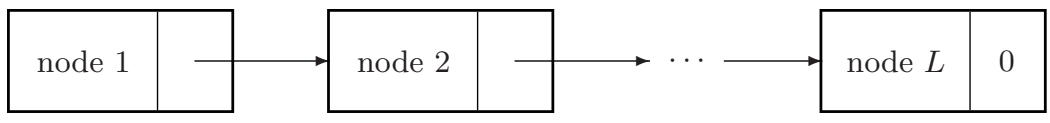

Fig. 1. Structure of the one-way linked list memory allocation for the series, the divisors and the arbitrary length coefficients of L nodes.

The series consists of a passport and a sequence of its terms connected in circular chain with one link (Figure 2). The series passport contains its general characteristics: the number of terms in the series $(N)$ and the link on its last term $(L A S T)$.

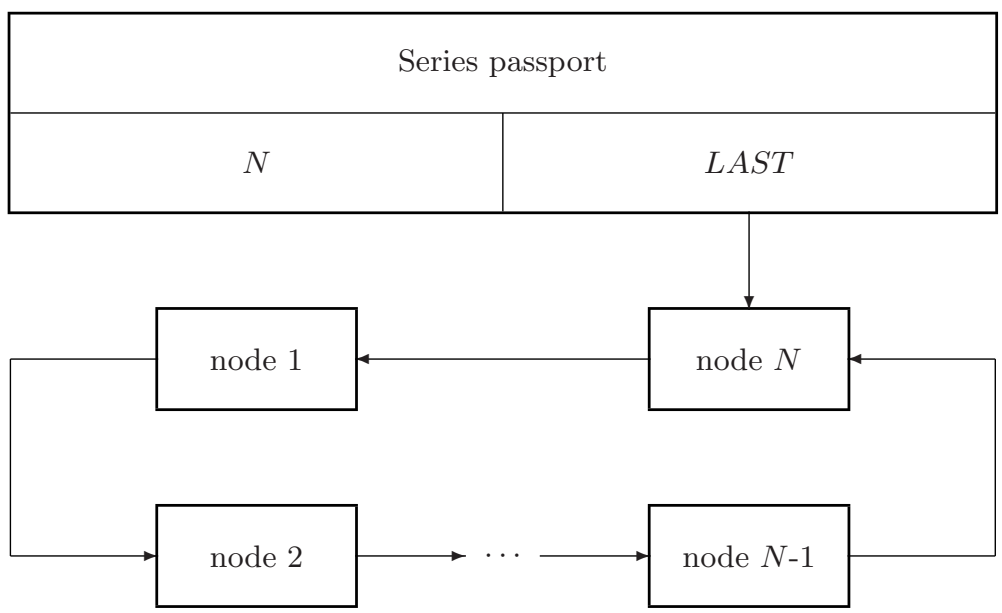

Fig. 2. Structure of a series 
The node is an elementary structure unit. It includes the main characteristics of the separate term of a series (Figure 3):

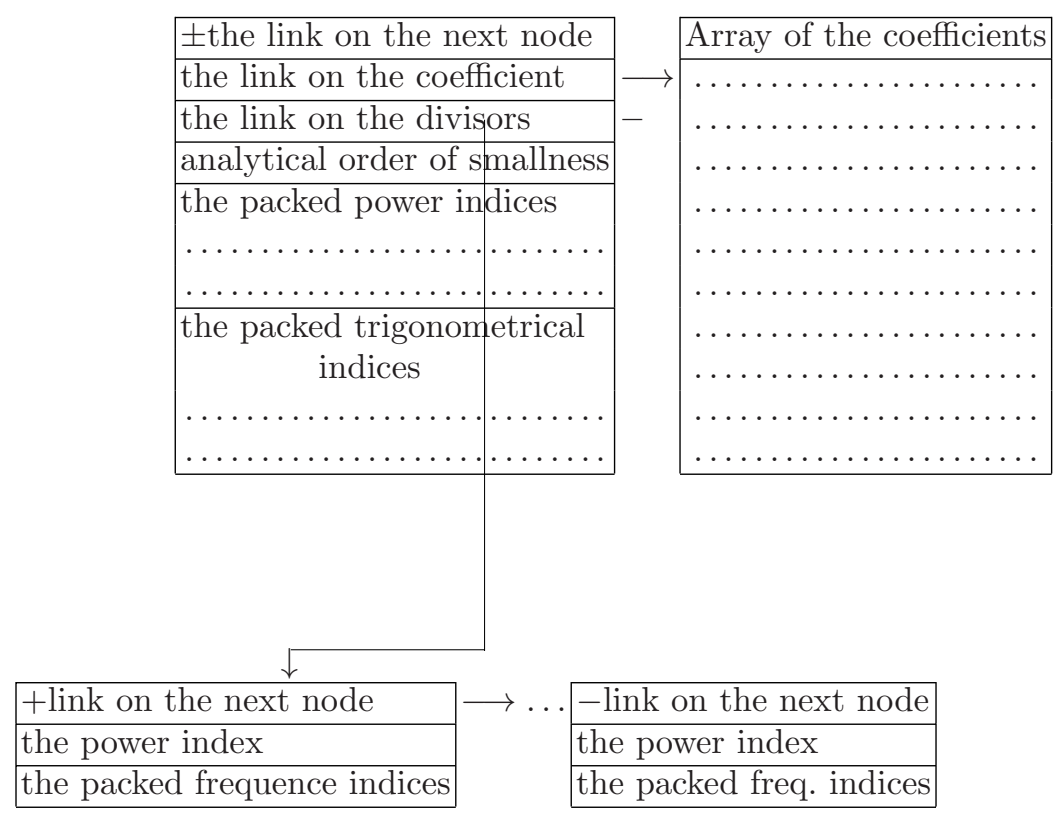

Fig. 3. Structure of a node.

* the link on the next node;

* the name of the trigonometric function defined by the sign ' + ' for a cosine term and the sign '-' for a sine term assigned to the above-mentioned link;

* the link on the beginning of the coefficient;

* the link on the beginning of the symbolic divisors or factors. If this link is equal to zero it means that this node does not contain any divisors;

* analytical order of smallness;

* the packed power and trigonometric indices.

Each term of the symbolic divisors or factors includes:

* the link on the next node;

* a flag assigned to the link and expressed by signs ' + ' or ' - '. It indicates whether the divisors are finished or not for the given node;

* an exponent index for one divisor. It can be positive or negative;

* the packed frequency indices.

In the case of rational coefficients, the double precision floating-point numbers for correct operations on integer numbers are used. If a numerator or a denominator of rational coefficient falls outside the limits of maximum permissible number then they are transformed automatically to floating-point coefficients and the processor continues its work. If the coefficients are of arbitrary length then they are represented in the form like to divisors (Figure 4). 


\begin{tabular}{|c|c|}
\hline+ link on the next node & -link on the next node \\
\hline numerator of rational number & numerator of rational number \\
\hline denominator of rational number & denominator of rational number \\
\hline
\end{tabular}

Fig. 4. Structure of an arbitrary length coefficient.

Each term of the coefficient of arbitrary length includes:

* the link on the next node;

* a flag assigned to the link and expressed by signs ' + ' or ' - '. It indicates whether the coefficient is finished or not for the given node;

* numerator of rational number;

* denominator of rational number.

The packed indices are able to occupy an arbitrary number of computer integer words dependent on the number of indices, the value of their range and the length of the computer integer word. All the operations performed on the nodes deal with the standard form of nodes. It means that the first trigonometric or frequency index not being zero, must be positive.

\section{Conclusion}

The Keplerian processor and analytical generator of special celestial mechanics functions based on PSPs are also proposed. They may be regarded as an illustration of the practical application of the processors. These systems are designed for implementing the expansions of the most important mathematical functions of celestial mechanics such as Legendre and Gegenbauer polynomials, Bessel and spherical functions, etc., for constructing the expansions of the elliptic motion functions of the unperturbed two-body problem and the expansions of the typical celestial mechanics functions such as Hansen coefficients, Newcomb operators, Kaula inclination functions and their generalizations, etc. The software of this sort is necessary to produce analytical solutions of various celestial mechanics problems.

PSPs are available on request in electronic form from itv@quasar.ipa.nw.ru.

\section{References}

1. Brumberg, V. A.: 1995, Analytical Techniques of Celestial Mechanics, Springer, Heidelberg.

2. Ivanova, T. V.: 1995, PSP: A New Poisson Series Processor, in: S. Ferraz-Mello, B. Morando and J.-E. Arlot (eds), Proceedings of the 172nd Sympozium of the International Astronomical Union, Dynamics, Ephemerides and Astrometry of the Solar System, Paris, France. Kluwer Academic Publishers.

3. Ivanova, T. V.: 2001, A New Echeloned Series Processor (EPSP), Celest. Mech. and Dyn. Astron., 80, Kluwer, 167-176.

4. Rom, A.: 1971, Echeloned Series Processor (ESP), Celest. Mech., 3, 331-345. 